\title{
Extramedullary Plasmacytoma of the Orbit as Initial Presentation of Multiple Myeloma
}

\author{
Rosniza Abdul Razak, ${ }^{1}$ Thomas Paulraj Thamboo, ${ }^{2}$ Shantha Amrith ${ }^{1}$ \\ ${ }^{1}$ Department of Ophthalmology, and ${ }^{2}$ Department of Pathology, National University Health \\ System, Singapore
}

\begin{abstract}
This report is of a patient with a rare extramedullary plasmacytoma of the orbit as the initial manifestation of systemic multiple myeloma. A 34-year-old otherwise healthy man presented with periorbital pain for 2 weeks with proptosis, diplopia, and limited ocular motility in the left eye. Magnetic resonance imaging showed an extraconal mass at the superotemporal region of the left orbit with bony erosion and an enhancing orbital lesion in the temporal quadrant of the right orbit. Histopathological examination of the orbital mass revealed a plasmacytoma with lambda light chain restriction. Systemic evaluation revealed an immunoglobulin $G$ myeloma with multiple myeloma in the first lumbar vertebra, hypercalcaemia, and renal impairment. Chemotherapy was planned. Plasmacytoma of the orbit is rare and can present as the first manifestation of multiple myeloma. Ophthalmologists should be aware of such a presentation and must act promptly. This report reviews the presenting features.
\end{abstract}

Key words: Multiple myeloma, Orbit, Plasmacytoma

Asian J Ophthalmol. 2011;13:24-6.

\section{Introduction}

Plasmacytoma is a descriptive term applied to tumours consisting of plasma cells..$^{1,2}$ Extramedullary plasmacytoma is a localized collection of plasma cells in an extraskeletal site. Extramedullary plasmacytomas are rare plasma cell tumours arising predominantly from submucosal tissue of the nasal cavity, paranasal sinuses, nasopharynx, oropharynx, and larynx., ${ }^{3,4}$ The incidence of plasmacytoma in the orbits is very rare. ${ }^{1,2,5,6}$ Orbital mass is a rare presenting feature in multiple myeloma.

\section{Case Report}

A 34-year-old otherwise healthy man from Malaysia presented in 2009 with periorbital pain for 2 weeks with proptosis, diplopia, and limited ocular motility in the left eye. Ophthalmic examination revealed his best-corrected visual acuity to be $6 / 6$ in both eyes. There was no relative afferent pupillary defect. There was 2-mm non-axial proptosis of the left eye with limited ocular motility and resistance to retropulsion. Diplopia was noted in almost all gazes except primary gaze. There was fullness of the left upper eyelid

Correspondence: Dr Shantha Amrith, Department of Ophthalmology, National University Health System, 5 Lower Kent Ridge Road,

Singapore 119074 .

Tel: (65) 6772 5317;

E-mail: ophv14@nus.edu.sg
Figure 1. Fullness of the left upper eyelid with downward displacement of the eyeball.

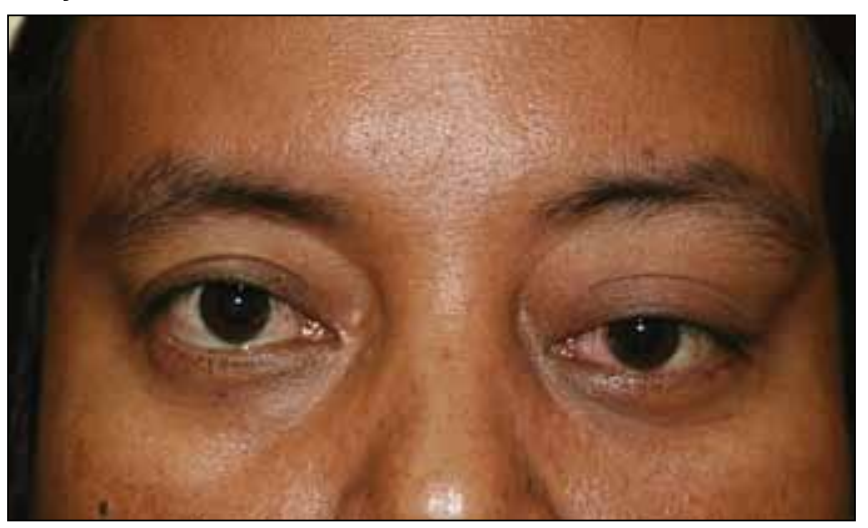

below the supraorbital rim (Figure 1). An ill-defined firm and mildly tender mass was palpable over the left superotemporal quadrant. Anterior segment examination of both eyes was unremarkable. Fundoscopy examination revealed superotemporal choroidal striae in the left eye. Systemic examination revealed no lymphadenopathy or hepatosplenomegaly.

Magnetic resonance imaging (MRI) showed an extraconal mass at the superotemporal region of the left eye, with the involvement of the adjacent bone. An enhancing orbital lesion was also noted in the medulla of the bone in the temporal quadrant of the right orbit (Figure 2). 
Figure 2. Magnetic resonance imaging scan. (a) Coronal T1-weighted with contrast showing an extraconal mass at the superotemporal region of the left orbit with bony involvement and adjacent dural thickening; and (b) axial T1-weighted showing an enhancing lesion in the medulla of the greater wing of the sphenoid on the right side (white arrow).

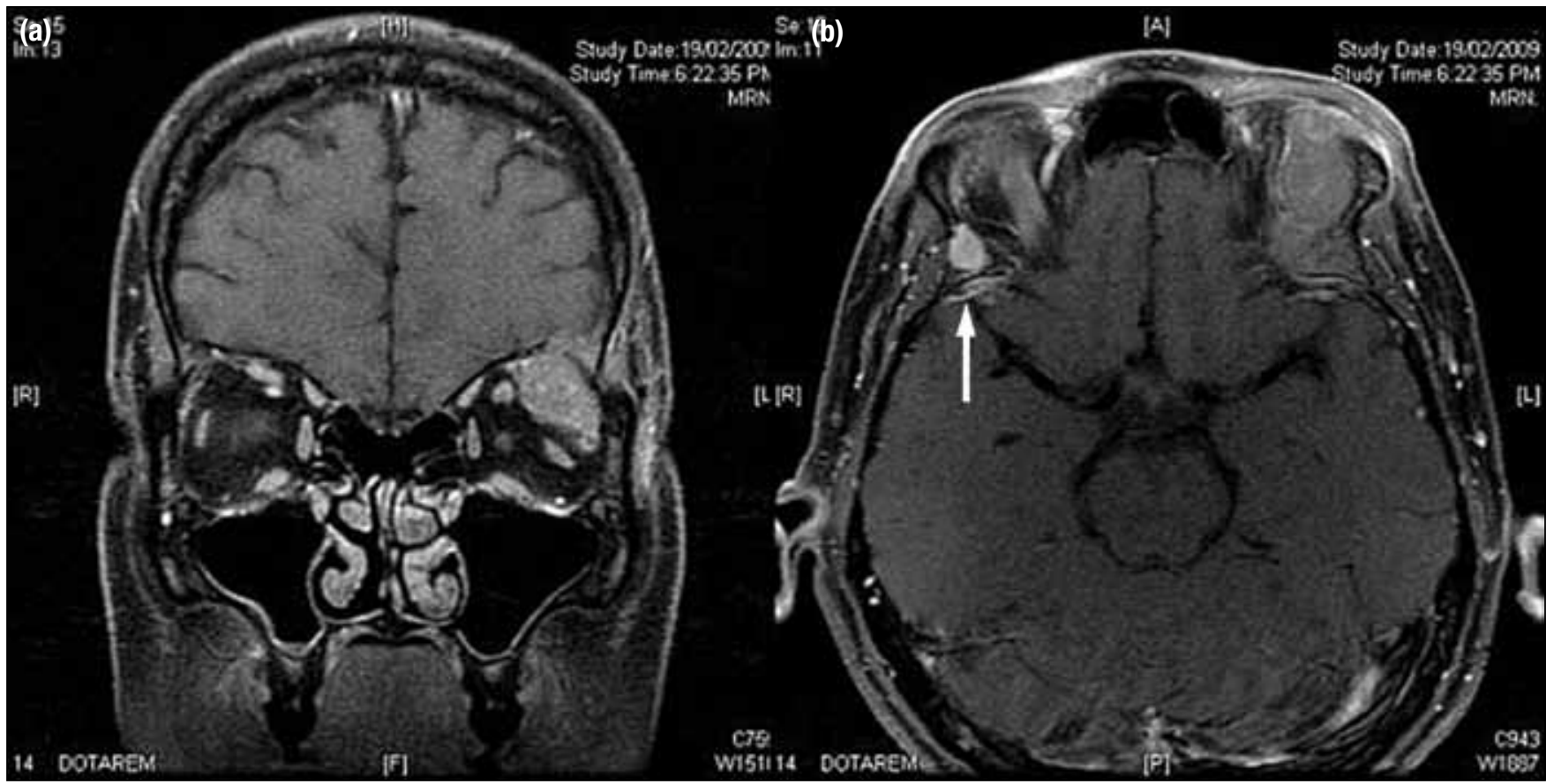

Figure 3. Histological examination of the orbital mass showing (a) sheets of plasma cells, many of which have atypical nuclei (haematoxylin and eosin stain; original magnification, $x$ 400); and (b) plasma cells with diffuse strong membranous staining for CD138 immunostain (original magnification, $x$ 400).

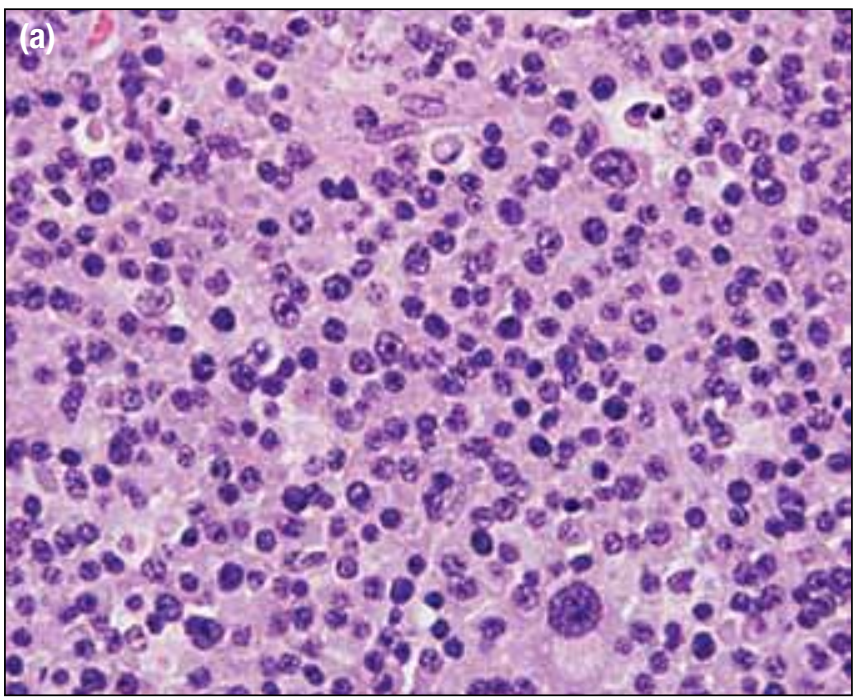

Anterior orbitotomy and tumour debulking was performed and histopathological examination of the orbital mass revealed a plasmacytoma with lambda light chain restriction (Figure 3). The patient was referred to the haematology and oncology teams for systemic evaluation, which revealed immunoglobulin G myeloma with multiple myeloma of the first lumbar vertebra (Figure 4), hypercalcaemia, and renal impairment. The patient was advised to have chemotherapy and he opted to have further treatment in his country of origin.

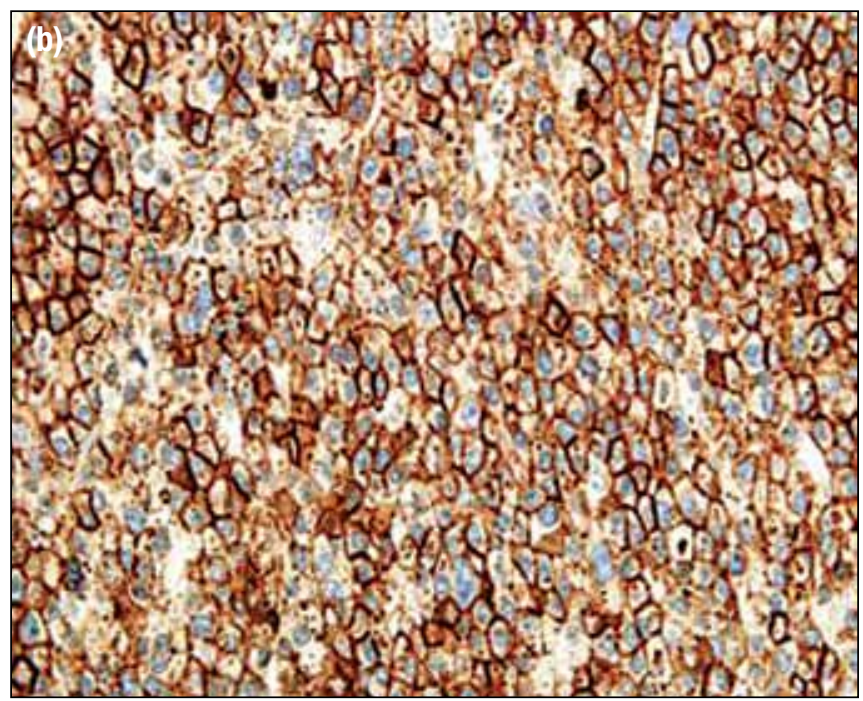

\section{Discussion}

Localised neoplastic proliferation of plasma cells in the bone marrow is known as solitary plasmacytoma of bone and, in soft tissues other than bone marrow, as extramedullary plasmacytoma. Plasma cell tumours of the orbit are uncommon, and can occur as solitary plasmacytoma of the soft tissues of the orbit or as part of systemic involvement of multiple myeloma. ${ }^{4-8}$ Solitary extramedullary plasmacytoma is a primary lesion and does not often metastasise. However, extramedullary plasmacytomas that 
Figure 4. Magnetic resonance imaging scan of the spine showing a lytic lesion in the first lumbar vertebra.

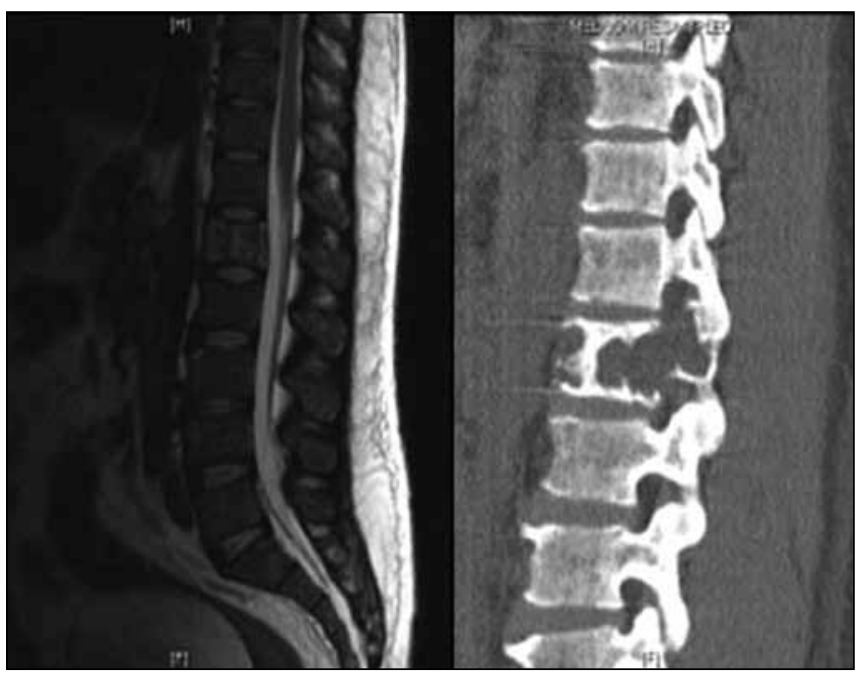

are manifestations of multiple myeloma are more aggressive with a worse clinical outcome. ${ }^{7}$

Patients usually present with progressive eyelid swelling, proptosis, and diplopia, as seen in this patient. More common conditions such as non-specific orbital inflammation, especially when patients present with pain, lymphoma, and tumour metastasis, should be considered in the differential diagnosis. In this patient, the MRI scan of the orbits revealed infiltration of bone marrow of the fellow orbit, thereby raising the suspicion of a haematological tumour. Diagnosis of plasmacytoma is made by histopathological examination of the orbital lesion. However, it is difficult to know whether the orbital lesion represents a solitary plasmacytoma until further investigations for systemic disease are done. Thus, comprehensive medical evaluation is necessary, including bone marrow biopsy, serum and urine immunoelectrophoresis, and skeletal survey to exclude systemic involvement. This patient presented with an orbital lesion, and systemic involvement was confirmed by laboratory evaluation and skeletal survey, and MRI confirmed the spinal involvement.

Plasmacytoma of the orbit can either present as an initial manifestation of multiple myeloma as in this patient or at the terminal stages as progression of the disease. The treatment options include radiotherapy, surgical removal, or chemotherapy and depend on the extent of the disease.

Extramedullary plasmacytoma is a rare condition and can present initially as an orbital mass. It is important for ophthalmologists to realise that not all extramedullary plasmacytomas are solitary and there is a need for systemic investigations for multiple myeloma, which carries a poorer prognosis.

\section{References}

1. Adkins JW, Shields JA, Shields CL, Eagle RC Jr, Flanagan JC, Campanella PC. Plasmacytoma of the eye and orbit. Int Ophthalmol. 1997;20:339-43

2. Agrawal PK, Mittal S, Gupta P, Agrawal K, Agrawal PK. Plasmacytoma of orbit. Indian J Ophthalmol. 1993;41:34-6.

3. Megat Shiraz MA, Jong YH, Primuharsa SH. Extramedullary plasmacytoma in the maxillary sinus. Singapore Med J. 2008;49: e310-1.

4. Uceda-Montanes A, Blanco G, Saornil MA, Gonzalez C, Sarasa $\mathrm{JL}$, Cueves L. Extramedullary plasmacytoma of the orbit. Acta Ophthalmol Scand. 2000:78:601-3

5. Knecht P, Schuler R, Chaloupka K. Rapid progressive extramedullary plasmacytoma in the orbit. Klin Monatsbl Augenheilkd. 2008; 225:514-6.

6. Sharma A, Kaushal M, Chaturvedi NK, Yadav R. Cytodiagnosis of multiple myeloma presenting as orbital involvement: a case report. CytoJournal. 2006;10:3-19.

7. Soutar R, Lucraft H, Jackson G, et al. United Kingdom Myeloma Forum; guidelines on the diagnosis and management of solitary plasmacytoma of bone and solitary extramedullary plasmacytoma. Br J Haematol. 2004;124:717-26.

8. Lazaridou MN, Micallef-Eynaud P, Hanna IT. Soft tissue plasmacytoma of the orbit as part of the spectrum of multiple myeloma. Orbit. 2007;26:315-8. 